Revue d'histoire de l'Amérique française

REVUE D.HISTOIRE DE L'AMÉRIQUE FRANÇAISE

\title{
Les Corte-Real et le Nouveau-Monde (suite)
}

\section{Eduardo Brazão}

Volume 19, numéro 2, septembre 1965

URI : https://id.erudit.org/iderudit/302464ar

DOI : https://doi.org/10.7202/302464ar

Aller au sommaire du numéro

Éditeur(s)

Institut d'histoire de l'Amérique française

ISSN

0035-2357 (imprimé)

1492-1383 (numérique)

Découvrir la revue

Citer cet article

Brazão, E. (1965). Les Corte-Real et le Nouveau-Monde (suite). Revue d'histoire de l'Amérique française, 19(2), 163-202. https://doi.org/10.7202/302464ar d'utilisation que vous pouvez consulter en ligne.

https://apropos.erudit.org/fr/usagers/politique-dutilisation/ 


\title{
LES CORTE-REAL ET LE NOUVEAU-MONDE * (suite)
}

\author{
II \\ LES VOYAGES DE GASPAR CORTE-REAL \\ DANS L'ATLANTIQUE-NORD
}

Henry Harrisse écrit ${ }^{1}$ : “ . . les Portugais méditaient, vingt ans au moins avant le célèbre voyage de Christophe Colomb, de franchir l'Océan Atlantique dans la direction de l'ouest". Plus loin, il précise davantage sa pensée: "On peut même faire remonter aux premières années de la seconde moitié du $\mathrm{XV}^{\mathbf{e}}$ siècle des projets, voire des tentatives, dans cette direction."

De son côté, Morison affirme: "Why should anyone assume in 1452 that the nine Azores exhausted the islands of the North Atlantic ? Corvo and Flores were discovered after the other seven; why should there not be more still? There was every reason for the enquiring maritime mind of that era to suppose that more could be found. It is a matter of historical record that such searches were made." ${ }^{2}$

Peut-être la première de ces tentatives était-elle celle de Diogo de Teive, écuyer de l'infant dom Henri ${ }^{3}$ qui aurait, en 1452, découvert les îles de Corvo et Flores ${ }^{4}$ et, poussant son exploration plus à l'ouest, aurait aperçu la côte du NouveauMonde à la hauteur de Terre-Neuve. Jaime Cortesão ${ }^{5}$, qui s'est tout particulièrement intéressé à ce présumé voyage, l'a reconstitué à l'aide des descriptions de Fernando Colomb, d'après les notes de son père et des dépositions de trois témoins entendus au cours du procès que Diego Colomb intenta à la Couronne de Castille, entre 1532 et 1535.

${ }^{*}$ Voir notre Revue, XIX: 3-52. 
Dans l'histoire apologétique des annales de Christophe Colomb ${ }^{6}$, dont l'original est disparu, mais que l'évêque Bartolomeu de las Casas a reproduit en partie dans sa fameuse Historia de la Indias ${ }^{7}$, le fils du navigateur aurait écrit:

Un dénommé Diogo de Teive se lança également à la recherche de cette île (l'île légendaire des Sept Cités) ${ }^{8}$. Son pilote, un certain Pedro de Velasco, originaire de Palos de Moguer, au Portugal ${ }^{9}$, fit à l'amiral, à Santa Maria de Arrabida, le récit suivant: Partis de Fayal, ils parcoururent plus de 150 lieues en direction du sud-ouest ${ }^{10}$. Au retour, ils découvrirent l'île Flores vers laquelle les guidaient les nombreux oiseaux volant dans cette direction. Comme il s'agissait d'oiseaux terrestres et non marins, on crut qu'ils viendraient à se poser sur une terre quelconque. Ensuite, ils s'acheminèrent tellement vers le nord-ouest ${ }^{11}$ qu'ils arrivèrent au cap Clear, en Irlande, par l'Est ${ }^{12}$. Ils y rencontrèrent les forts vents de Poente, mais la mer était calme. Ils en conclurent à la proximité d'une terre qui les séparait de l'Occident. Comme c'était le mois d'août, ils n'osèrent retourner à l'île par crainte d'y être surpris par l'hiver ${ }^{13}$. Cela se passait plus de quarante années avant la découverte de nos Indes ${ }^{14}$ et lui fut confirmé par le récit d'un marin du port de Santa Maria. Celui-ci racontait que, lors d'un voyage en Irlande, en route vers l'Occident, il aperçut cette terre, qu'il croyait alors appartenir à la Tartarie. C'est probablement celle que nous appelons terre des Morues où les tempêtes les empêchaient de s'approcher. Le Galicien Pedro de Velasco abonde dans le même sens lorsqu'il affirme, à Murcie, en Castille, qu'en route vers l'Irlande, ils poussèrent tellement vers le nord-ouest qu'ils atteignirent une terre à l'ouest de l'Irlande. Il la prit pour celle qu'un nommé Fernão Dulano ${ }^{15}$ tenta de découvrir. Je le raconterai très fidèlement, comme je l'ai lu dans les écrits de mon père, parce que l'on savait tirer des conséquences démesurées de petites causes.

Ces commentaires douteux du fils de Colomb ont cependant fait l'objet d'une juste appréciation de la part de Jaime Cortesão ${ }^{16}$ : 
D'après les déclarations de Fernando Colomb, ces faits importants proviennent des notes manuscrites de l'Amiral. L'examen des autres renseignements puisés dans ce même chapitre (dont le manuscrit original est également inconnu) nous amène à la même conclusion: tous se rapportent à des personnages connus: ils mentionnent des circonstances que l'on a toutes les raisons de croire vraies ou tout au moins plausibles, ce qui accorde une grande crédibilité au tout. Mais l'œuvre du fils de l'Amiral avait un but unique: exalter la mémoire paternelle. Les renseignements révélés étaient de nature à ternir la mémoire de Colomb comme découvreur de l'Amérique. Dans notre étude sur les découvertes précolombiennes des Portugais, nous avons prouvé que tous les efforts du fils de l'Amiral tendaient à diminuer la valeur des notes paternelles à ce sujet, car il s'est abaissé, sans aucun doute, à inventer, falsifier et même mutiler sciemment certains textes. Dans notre propre étude, nous ne faisons que suivre les traces d'Altolaguirre y Duval dans son œuvre Cristobal Colon y Pablo del Pozzo Toscanelli (Madrid, 1903) où l'illustre historien espagnol prouve abondamment que Fernando Colomb dénatura fréquemment les faits, et cela, chaque fois que ceux-ci pouvaient porter atteinte à la mémoire paternelle.

D'autre part, les preuves établies lors de certains procès qui ont eu lieu entre 1532 et 1535 , et publiées par Cesário Fernandez Duro ${ }^{17}$, présentent une coïncidence avec les renseignements donnés par le fils du "Grand Amiral", même si nous $\mathrm{y}$ ajoutons peu de foi.

Si, d'une part, on comprend le silence entourant une telle exploration, plusieurs hésitent à le relier au choix que Teive avait fait d'un Espagnol d'Andalousie comme pilote. Il s'agit de Pedro de Velasco, dans le récit colombien, ou de Pero Vasquez de la Frontera, tous deux de Palos, que certains croient être une seule et même personne ${ }^{18}$. Dans l'exploration et la découverte d'un monde inconnu, on partageait ainsi le secret avec un étranger et avec des pays rivaux. L'explication de Cortesão n'en est pas moins acceptable, jusqu'à un certain point ${ }^{19}$ : entre nos côtes de l'Algarve, bases principales, on le sait, de la navigation 
d'Henri, et les ports andalous les plus proches, il existait depuis longtemps des relations sociales étroites "dans le domaine de leur vie maritime et de leur culture respectives". Selon les généalogistes, Diogo de Teive était lui-même marié à Maria Gonçalves de Vargas e Gusmão, fille de Martins Gonçalves de Vargas, gentilhomme castillan de Séville ${ }^{20}$. Comme nous l'avons déjà dit, notre explication est différente : il suffit de considérer le fait que les hommes de cette époque ne concevaient pas le patriotisme tel que nous l'entendons aujourd'hui: ils servaient seulement le prince qui les engageait, avec la même loyauté que nous accordons aujourd'hui à la nation et à l'État auxquels nous appartenons. L'argument du silence, si souvent invoqué, n'est pas pour autant infirmé ni affecté par cette collaboration étrangère.

Si nous admettons, avec Cortesão, une erreur dans le texte de Las Casas, la première de nos tentatives pour découvrir les terres des épices par l'Occident serait peut-être celle de Teive. Nous nous inscrivons en faux lorsque Las Casas affirme, reprenant la thèse de Fernando Colomb, avoir suivi le navire de Teive en direction nord-est. L'illustre auteur trouve absurde la route indiquée puisque l'on prétendait aller à la découverte d'Antilia, que l'on croyait toujours à l'Ouest ${ }^{21}$. A ce sujet, il formule des commentaires judicieux:

Il restait à tenter de reconstituer le texte primitif le plus exactement possible d'après les versions de Fernando Colomb et de Las Casas, travail d'autant plus ardu que ces deux versions diffèrent en partie et reproduisent les mêmes erreurs. Nous avons commencé, en conséquence, par démontrer que la phrase "camminarono tanto per Nordeste che presero il capo di Chiara in Irlanda per Loeste" devrait se lire: ils ont tellement navigué en direction du nord-ouest que, pour eux, le cap Clear se trouvait à l'Est. La version originale est en effet absurde. De fait, il est impossible de naviguer vers le nord-est et d'y trouver une île, Antilha ou l'île des Sept Cités que, selon ce même Fernando Colomb, les navigateurs devaient trouver à l'ouest de Madère et des Açores. Il était encore moins possible, en 
laissant Fayal, soit en direction du nord-est, ou soit en direction du cap Clear même, en Irlande, de découvrir ou de tenter de découvrir des terres, à l'est de ce cap, qui ne fussent déjà connues des navigateurs. Fernando Colomb lui-même, identifiant la région découverte ou supposée être la Terre des Morues, prétendait que le voyage s'était fait en direction du nord-ouest et non du nord-est. Il ne subsiste aucun doute à ce sujet: Colomb a signalé, dans ses notes, les voyages de Diogo de Teive dans deux directions: vers le sud-ouest (libecchio) où il découvrit, au retour, les îles Flores et Corvo; et vers le nord-ouest. C'est à la fin de ce voyage que les navigateurs trouvèrent des indices sûrs de l'existence d'une terre. Le phénomène, mentionné par le navigateur, de vents violents sur une mer calme, annonçait la proximité de la terre, et les marins portugais le mentionnaient souvent lorsque, revenant de la côte occidentale d'Afrique, ils rencontraient les alizés du nord-est au sud de Madère.

Morison, comme presque toujours, n'est pas d'accord avec la thèse portugaise ${ }^{22}$ quand il affirme: "They (Teive e Velasco) probably discovered Corvo and Flores in the Azores; they certainly discovered nothing else." ${ }^{23}$

Viennent ensuite de nombreux actes de concession royale visant des terres à découvrir dans ces parages. En même temps l'on tentait (ce qui nous semble exact) de se rapprocher de ce que l'on supposait être l'extrémité occidentale du monde décrit par Marco Polo. Il était normal qu'il en fût ainsi, après tout ce que nous avons signalé. L'infant Henri et tous ceux qui, au Portugal, dirigeaient nos explorations devaient nécessairement tenter de se diriger vers le nord-ouest au fur et à mesure que progressait le périple africain. Les Açores furent en quelque sorte une catapulte naturelle vers les côtes américaines. Dans une publication récente, Frank Debenham ${ }^{24}$ écrivait: “... the Azores might well have become a starting point for the rediscovery of America..." Et Jaime Cortesão, historien de grande valeur, spécialisé dans ce chapitre de l'histoire du Portugal, affirmait ${ }^{25}$ : "Nous n'oublions pas que la colonisation des Açores fut la véritable école où les Portugais ont appris la navi- 
gation hauturière et au long cours et où se sont formés les maîtres intrépides de cet art."

Nous avions appris l'existence de terres par-delà l'Atlantique, d'abord par les sagas, et plus tard par la littérature et les récits, véridiques ou non, de cette époque. Et, au même temps, c'est là, dans ces îles, découvertes et peuplées par nous, que sont apparues les premières preuves de cette existence: les courants échouaient sur les plages des troncs d'arbres, inconnus dans ces régions, et creusés avec des instruments qui n'étaient pas de fer, et même des cadavres à la figure plus large et différente de celle des chrétiens ${ }^{\mathbf{2}}$.

Nous énumérons ici d'autres preuves, si on peut les considérer comme telles, de notre intention d'explorer le nord de l'Atlantique, et même de nos succès possibles ${ }^{27}$ :

La donation d'Alphonse V à l'Infant Ferdinand, son frère, en date du 10 décembre 1457, "de quaesquer ilhas, que depois desta data se acharem" (de quelques îles qu'on découvrirait après cette date).

La donation royale du 19 février 1462 à João Vogado de deux îles "segundo a carta de marear (...) chamadas, uma a ilha do Lovo et a outra Capraria" (suivant la carte marine (...) appelées, l'une Lovo et l'autre Capraria) ${ }^{28}$. Duarte Leite les croit fictives, même si elles figurent sur les cartes des $X V^{e}$ et $\mathrm{XVI} \mathrm{e}^{\mathrm{e}}$ siècles, dans un groupe méridional du supposé archipel açoréen ${ }^{29}$.

Le 29 octobre de cette même année 1462, on trouve une autre donation à l'Infant Ferdinand: "Em Lisboa... faz o mesmo rei merce ao dito infante D. Fernando, de uma ilha, que Gonçalo Fernandes, de Tavira, diz que vira vindo das pescarias do rio de Ouro, a oes-nor-oeste das Canárias e da Madeira, e a que se não pudera chegar por o tempo ser contrario. Esta doação teria efeito em qualquer tempo em que tal ilha se achasse, ou por navios do mesmo infante, ou pelos de qualquer outra pessoa" (A Lisbonne... ce même roi fait don audit infant Ferdinand, d'une île que Gonçalo Fernandes, de Tavira, 
Dans la donation du 10 novembre, le roi portugais ajoutait que, du fait de la concession antérieure, il s'agissait d'îles désertes

que a dito Fernão Teles por si ou por outrem mande povoar, e poderia ser que, em ele as assi mandando buscar, seus navios ou gente achariam as Sete Cidades ou algumas outras ilhas povoadas... declaro por esta minha carta que a minha tenção foi, logo ao tempo que lhas assi dei, de assi se entender a dita mercê a ilhas povoadas como nom povoadas.

("que ledit Fernão Teles, pour lui-même ou pour un autre, envoya peupler, et il se pourrait également, en les envoyant à leur recherche, que ses navires et ses gens atteignent les Sept Cités ou d'autres îles habitées... je déclare par cette lettre que mon intention est de lui donner ces îles, qu'elles soient peuplées ou non").

L'île des Sept Cités ${ }^{36}$, appelée antérieurement Antilia, était, on le sait, une île légendaire peuplée de chrétiens. Sept évêques et quelques fidèles y seraient débarqués, fuyant la péninsule ibérique durant les invasions arabes du VIII e siècle. Mais la légende devenait peu à peu réalité: la fameuse île changeait d'emplacement à chaque découverte que l'on faisait vers l'Occident à la recherche des Sept Cités. C'était une espèce de mirage qui nous attirait vers les côtes du Nouveau-Monde! Aux Açores, le nom devait s'identifier à la toponymie locale en rappelant les actes de navigateurs d'origines aussi diverses. Mais Fernão Teles qui, nous dit Zurara dans la Crónica do Conde D. Pedro de Menezes, était un homme à l'esprit pratique et soucieux de ses intérêts matériels, n'allait pas se lancer dans n'importe quelle aventure. Sa seconde lettre patente apporte une rectification qui représente plus que de l'imagination.

Cortesão ${ }^{37}$ relie très objectivement cette expédition possible de Fernão Teles (en 1476, suivant ses calculs) à celles de Teive et de João Vaz (celle-ci, naturellement, devra être incluse dans la liste que nous présentons). Voyons, une fois de plus, ce que dit l'éminent historien de nos découvertes: 
L'expédition de Teive suppose une œuvre de continuité, confirmée par ailleurs dans les notes de Colomb, et conséquemment la connaissance postérieure d'un vaste continent s'étendant vers le Nord. Cela expliquerait assez bien la demande, au roi de Danemark, de la collaboration de marins scandinaves, plus expérimentés sur ces mers, et l'objectif d'une expédition relative aux terres continentales. D'autre part, avant de poursuivre l'expédition vers les mers du Nord, la prudence la plus élémentaire exigeait d'affermir la possession des terres déjà connues dans cette région, pour prévenir une continuation du voyage jusque vers ces terres et la possibilité d'un conflit de souveraineté avec le roi de Danemark. Ces deux facteurs se complètent, pour ainsi dire. Le prince, une fois convaincu de la nécessité de cette mesure préventive, la meilleure façon pour lui de résoudre ce problème délicat sera de garantir au navigateur ou commanditaire luimême la possession des terres déjà connues, afin de l'intéresser à l'expédition luso-scandinave, à la recherche d'autres nouvelles terres. Ce qui rend cette hypothèse plus acceptable est l'achat, par Teles, des îles Flores, et l'objectif des Sept Cités, déclaré dans les lettres de donation, en sachant bien où elles se trouvent comme nous en avons aujourd'hui la preuve. Tel nous semble être le fil qui relie entre elles l'expédition de Teive, celle de Teles et celle des mers du Nord.

Et plus loin:

... Nous sommes convaincus que cette appréciation des faits est infiniment plus cohérente et plus convaincante que les avancés de Larsen. Nous savons que sur la carte de Cantino (1502), le Groenland était indiqué comme étant l'extrémité de l'Asie ${ }^{38}$, et que, selon Fernando Colomb, les découvreurs des terres des morues le confondirent avec une partie de la Tartarie marquant le retour vers l'Occident. On comprend mieux que l'infant dom Henri, ou du moins le prince Jean aient voulu savoir s'il serait possible d'atteindre l'Inde par cette route. Cette entreprise a eu pour effet: de confirmer l'existence de terres, dans ces régions, et de convaincre des 
difficultés excessives que les glaces opposent aux voyages par le Nord.

Nous avons ensuite la donation, en date du 30 juin 1484, à Fernão Domingos do Arco de "uma ilha que ia buscar" ("d'une île à aller découvrir"). Et dans ces mêmes sens et objectif, toujours l'île des Sept Cités, la concession du 4 août 1486 au Flamand d'Ulmo, capitaine de l'île Terceira ${ }^{39}$ : "Queria dar achada huma grande ylha ou ylhas, ou terra firme per costa, que se presume ser a ylha das Sete cidades... a sua propria custa" ("Il désire aller découvrir une grande île, ou des îles, ou terre ferme, près des côtes, que l'on croit être l'île aux Sept Cités... à ses propres frais"). On trouve cette citation dans les lettres patentes qui lui étaient adressées. Le 24 juillet et le 4 août de cette même année furent signées les lettres confirmant le contrat entre Ulmo ${ }^{40}$ et João Afonso de Estreito par lequel celui-ci cédait à celui-là la moitié de ses droits de concession ${ }^{41}$.

Alvaro et João da Fonte, de Sainte-Marie des Açores, s'étaient aussi lancés dans cette entreprise de navigation vers l'Ouest. Frutuoso le souligne ainsi, en se référant aux deux navigateurs: "que gastaram toda a sua fazenda no descobrimento da ilha nova, sem a poder achar" ("qui dépensèrent tout leur argent à la découverte de l'île nouvelle sans jamais la trouver").

Le 28 octobre 1499, le roi Manuel, par lettres patentes, concédait à João Fernandes, le Labrador (laboureur), la capitainerie "de qualquer ilha ou ilhas, asy provoadas como despovoadas, que elle descobrill e achar novamente" ("de toute île ou îles, peuplées ou non, qu'il découvrirait ou trouverait à nouveau"). Ces mots, ajoutés aux autres preuves, nous portent à conclure que notre Açoréen de Terceira avait déjà découvert ces terres, que nous avons appelées ensuite: terres de Corte-Real ${ }^{42}$.

Nous arrivons enfin à la lettre de donation à Gaspar CorteReal, en l'an 1500, qui nous révèle en même temps les tentatives déjà faites par ce fameux navigateur portugais: 
...fazemos saber, que por quanto Gaspar Corte, rreall, fidalguo da nosa (casa), os dias pasados, se trabalhou per sy e à sua custa, com navyos e homes, de buscar e descubrir e achar com muyto seu trabalho e despesa de sua fazenda e peryguo de sua pesoa, algumas ilhas e terra firme, e pelo comsyguynte o quer ainda agora conthenuar e por em hobra e fazer niso quamto poder por achar as ditas ilhas e terra...

("...nous faisons savoir, en ce qui a trait à Gaspar Corte Real, gentilhomme de notre (maison) que, ces jours passés, il a travaillé, lui-même et à ses frais, avec des navires et des hommes, à chercher et découvrir et trouver avec beaucoup de travail et de dépenses de son argent et au péril de sa personne, quelques îles et de la terre ferme, et par la suite, il désire continuer et mettre en œuvre et faire à ce sujet tout en son pouvoir pour trouver lesdites îles et terre...")

Gaspar Corte-Real était le plus jeune fils de João Vaz et de sa femme, Maria de Abarca, cette belle Galicienne qui, nous l'avons déjà signalé à la suite de certains généalogistes, aurait été enlevée de sa maison paternelle, dans la ville de Tuy, par le fougueux gentilhomme d'Algarve. L'aîné des descendants était Vasco Anes, suivi de Miguel et de trois filles: Joana, qui épousa Guilherme Moniz Barreto; Eyria, qui devint la femme de Pedro Goes da Silva qui l'assassina; et Isabelle, mariée à Jobst de Hürter, que nous avons appelé d'Utra et qui succéda à son père à la capitainerie de l'île Fayal ${ }^{43}$.

Henry Harrisse qui, aidé du chercheur açoréen Ernesto do Canto, consacra beaucoup d'efforts à scruter la vie et les exploits des navigateurs Corte-Real, en vint à la conclusion que Gaspar serait né vers 1450 . On ne sait rien de sa jeunesse, si ce n'est ce que révèle Damião de Góis: qu'il servait le roi Manuel alors qu'il était encore duc de Beja: “... cujo criado já fora em sendo Duque de Beja" (dont il avait été le serviteur quand il était duc de Beja). Cependant, même s'il était troisième fils, 
il était très lié au monarque qui l'estimait et le tenait en grande estime, comme en témoignent les faveurs et les égards qu'il en a reçus. Rappelons-nous ce passage des lettres patentes à son frère Miguel, en date du 15 janvier $1502^{44}$, où le roi disait:

...e asy aos muytos serviços que temos recebido, e as diamte esperamos reçeber do dito Miguel Corte Reall, que seemdo casso que elle nom ache o dito sseu irmaão (Gaspar), ou semdo falecido, o que Deus nam mande...

("...et ainsi aux nombreux services que nous avons reçus et que nous nous attendons de recevoir à l'avenir du dit Miguel Corte Real, qui, au cas où celui-ci ne trouverait pas son dit frère (Gaspar) ou au cas de sa mort, qu'il ne plaise à Dieu ...”). Mais en plus de cela, le cadet des Corte-Real, à cette époque où le droit de primogéniture était à l'honneur, réussit à prendre bientôt possession des importantes propriétés de l'île Terceira que son père avait arbitrairement enlevées à son propriétaire légitime, José Leonardes. Celui-ci les avait acquises légalement, cinq ans auparavant, de Diogo de Teive, représentant légal de Jácome de Bruges, propriétaire de l'île. Après la disparition de Gaspar, en 1503, ces "terres incultes" sont revenues, par un jugement du tribunal, à la famille qui en avait été dépouillée.

Très tôt, croit-on, il dut se rendre à Terceira; nous le retrouvons donc à Angra, lieutenant de João Vaz jusqu'à la mort de celui-ci, le 3 février $1496{ }^{45}$, et ensuite, lieutenant de son frère aîné, Vasco Anes; comme le dit Harrisse ${ }^{46}$ : de la même façon que Diogo de Teive avait gouverné l'île entière pour Jácome de Bruges.

C'est ici, à cette véritable école de navigateurs, que Gaspar Corte-Real se prépara à sa fameuse entreprise vers l'Ouest. Il devait posséder tous les renseignements possibles (et il y en avait !) que les pêcheurs de l'archipel et de cette île en particulier lui avaient apportés des terres du Nord et du NordOuest qu'ils avaient visitées. Ces gens connaissaient déjà le Groenland et la côte limite de l'Occident. Rappelons les tenta- 
tives, déjà signalées, toujours à la recherche des Sept Cités, et surtout le voyage de João Fernandes et de Pero de Barcelos qui ont dû hiverner, deux fois peut-être, de 1491 au début de 1495, au sud de Terre-Neuve, possiblement au Cap Breton, découvrant ce qui devait s'appeler plus tard le golfe Saint-Laurent. D'après nous, et au point où en sont les recherches à ce sujet, ils furent les premiers à redécouvrir la Terre Neuve des Temps Modernes, quoique Gaspar Corte-Real soit considéré comme son vrai découvreur, car nous avons des preuves écrites de son second voyage ${ }^{47}$. On sait également de façon certaine qu'il fut le premier à débarquer sur ces terres qui prirent plus tard le nom de son illustre famille.

Or, à cette époque, la prise de possession était indispensable à la propriété réelle outre-mer. A cette fin, nos navigateurs laissaient des marques de pierre, avec nos armes, aux endroits qu'ils découvraient et rattachaient à la couronne du Portugal. Les cartographes ont remplacé ces armes par des drapeaux sur les cartes anciennes ${ }^{47 \mathrm{a}}$.

Ramusio écrit: "Il primo (per quel che si sa) fu Gasparo Cortereale Portoghese ${ }^{48}$..." L'Espagnol Lopes de Gomara est également très clair lorsqu'il affirme: "Fuè allà Gaspar Cortes Reales, el año de 1500, con dos carabelas. No hallò el estrecho que buscaba. Dejò su nombre à las islas que estám à la boca del golfo Cuadrado y en mas de cincuenta grados..." ${ }^{49}$. Son compatriote Antonio Herrera, chroniqueur royal, a reproduit ce texte presque intégralement ${ }^{50}$. Et Jeronimo Osório, un de nos grands humanistes, a laissé cette inscription lapidaire: "Rursus anno MDI se in eamdem regionem contulit ut latius littora illius omnia pervagaretur et gentis mores et instituta perdisceret. Sed quid illi acciderit, aut quo fato absumptus fuerit, nunquam scire potuit" ${ }^{51}$. Antonio Galvão, dans son fameux Tratado... dos diversos e desvayrados caminhos... écrit: "Neste mesmo anno de 500 diz que pedio Gaspar Corte real Licença a el rey dom Manuel pera yr descobrir a terra Noua..."52 (Cette même année 1500, Gaspar Corte-Real demanda au roi dom Manuel l'autorisation d'aller découvrir la 
terre Nouvelle...). D'aller découvrir la terre Nouvelle doit se rendre, en langue moderne, par: aller prendre possession de cette île, peut-être même l'île des "Sept Cités", que nous avions déjà trouvée et découverte. João Fernandes, revenant du Groenland, l'avait déjà aperçue; il en avait remarqué les contours en passant près de ses rives et de ses échancrures. Nous restons donc sur cette position, jusqu'à preuve complète du contraire.

Mais Gaspar Corte-Real avait près de 50 ans au moment de ce voyage que les lettres patentes de 1500 autorisaient. A cette époque, on vieillissait beaucoup plus rapidement qu'aujourd'hui, et cet âge était considéré alors comme assez avancé. Serait-il possible qu'un noble portugais du $\mathrm{XV}^{\mathrm{e}}$ siècle, vivant dans cette ère extraordinaire de découvertes, où l'on distribuait honneurs et faveurs, eût commencé si tard le dur métier de navigateur ? Faudrait-il accepter l'hypothèse que ce jeune rejeton de l'impulsif João Vaz - "homem aventureiro, esforçado, \& desejoso de ganhar honrra" ("homme aventurier, courageux et désireux de remporter des honneurs") comme Damião de Góis ${ }^{53}$ décrit Gaspar, fût attiré par la mer alors que la voix des sirènes avait déjà perdu toute sa saveur? Ayant eu sous les yeux l'exemple de toute sa famille, y compris son père, si celui-ci était, vraiment, le découvreur du Nord, et placé dans une situation exceptionnelle pour se lancer dans l'aventure, vivant parmi des marins et des pêcheurs, serait-il possible ce retard dans l'exploration de la Mer inconnue?

Qui sait si ce n'est pas à une époque bien plus reculée que l'an 1500, que se rapportaient les lettres patentes du Roi Manuel, ses commencements de navigateur ${ }^{54}$ : “...os dias passados (Gaspar Corte Real), se trabalhou per sy e à sua custa, com navyos e homes, de buscar e descobrir e achar com muyto seu trabalho e despesa de sua fazenda e peryguo de sua pesoa, algumas ilhas e terra firme ..." ( . . . les jours passés, il (Gaspar Corte-Real) a travaillé lui-même et à ses frais, avec des navires et des hommes, à chercher et découvrir et trouver avec beaucoup de travail et de dépenses de son argent et au péril de sa personne, quelques îles et de la terre ferme). Et pourquoi pas 
en 1472, c'est-à-dire vingt-huit ans avant ? Gaspar Corte-Real aurait eu alors près de 22 ans. Ceci pourrait nous amener à l'hypothèse que Frutuoso, dans les Saudades da Terra, a confondu les faits, une fois de plus. S'il nomme João Vaz comme découvreur du Brésil, au lieu de Pedro Alvares Cabral, il peut également faire allusion à celui-là quand il devrait dire: "E vindo Gaspar Corte Real do descobrimento da Terra Nova dos Bacalhaus..." (Et GASPAR CORTE-REAL, revenant de découvrir la terre neuve des morues...). Ainsi serait complètement éclairci ce passage de la lettre du 17 septembre $1506^{55}$ au sujet de la transmission des droits de Gaspar à son frère Vasco Anes: "Pedimdonos ho dicto Vassqueanes Corte Reall por merçe, que por a dicta doaçam vir a traspassar a elle per fallecimento do dicto seu irmãao, segundo forma della, lhe mandassemos dar nossa carta de comfirmaçam em forma, \& visto por nos seu requerimento, \& avemdo respecto \& lembranca como ho dicto Gaspar Corte Reall, seu irmãao, foy o primeiro descubridor das dictas terras..." (Comme Vasco Anes Corte Reall nous a demandé que cette donation lui soit transférée, à cause de la mort de son frère, et que nous devons donner une lettre de confirmation, nous la lui accordons en hommage à la mémoire de Gaspar Corte Reall, son frère, LE PREMIER qui a découvert lesdites terres...).

Dans les lettres patentes de 1500 , le roi déclare que Gaspar, après son expérience coûteuse, déjà mentionnée, désirait "ainda comthenuar e por em hobra e fazer niso quamto poder por achar as ditas Ilhas e terras..." ("continuer encore et faire tout en son pouvoir pour trouver lesdites îles et terres..."). L'expression nous laisse clairement entendre, du moins d'après nous, que ces îles et la terre ferme étaient déjà connues. Il s'agit donc de l'autorisation royale conférée à Gaspar CorteReal de prendre possession de ces terres et îles au nom du monarque et également à son propre avantage car, à ce qu'on affirme, ses premières explorations au nord de l'Atlantique lui avaient coûté des sommes considérables (et à sa famille éga- 
lement). Par conséquent, le roi déclare qu'à cause de cela, il est digne de "toda a honra e merce e acreçemtamento", ("tous honneurs, faveurs et prospérité") en lui conçédant "em quaesquer ilhas ou terra firme que asy novamente achar ou descobrir, ele tenha e aja de nos, de juro e de herdade pera todo o sempre, as capytanias com as cousas seguintes..." ("quelques îles ou terre ferme qu'il pourrait trouver ou découvrir de nouveau, et qui seront à lui pour toujours ..."). Par conséquent, encore une fois on établit qu'il s'agissait de la simple possession de ce que Gaspar ou d'autres Portugais avaient déjà découvert: "novamente achar" ("découvert de nouveau"), comme il est écrit. Suivent les expressions notariales consacrées pour ces concessions royales. Le monarque réservait à la couronne, du reste, le droit de regard sur la façon dont Corte-Real administrerait lesdites terres qui lui appartiendraient plus tard.

Après avoir obtenu cette concession, Gaspar fit deux tentatives d'expédition. Damião de Góis et António Galvão ${ }^{56}$ font allusion à la première, qui ne donna aucun résultat appréciable parce qu'elle était trop au nord, se heurtant aux glaces du Groenland et peut-être à celles de la terre de Baffin actuelle ou du haut Labrador. La seconde expédition, qui suivit immédiatement, est signalée également par ces deux chroniqueurs, mais particulièrement par Alberto Cantino et Pedro Pasqualigo dans leurs lettres au duc de Ferrare et à la Seigneurie de Venise respectivement. Ces écrits datent du 17 et 18 octobre 1501 et renferment des descriptions de faits qui venaient tout juste d'être connus. Comme le signale Harrisse ${ }^{57}$, ils constituent aujourd'hui, avec la carte maritime portant le nom du premier, nos principales sources de référence au sujet du dernier voyage du plus jeune des Corte-Real. On ne peut évidemment avoir une idée très nette de la façon dont ses contemporains décrivaient ces entreprises. C'étaient des récits racontés peut-être par les marins eux-mêmes, mais touchant des événements survenus dans des régions encore inconnues ou mal délimitées sur les cartes. Ce n'était pas aussi simple qu'aujourd'hui où l'on n'aurait qu'à indiquer sur une mappe l'endroit où un événement s'est déroulé. 
Damião de Góis a probablement commencé à rédiger sa Crónica do Felicissimo Rei D. Manuel, à la demande du futur cardinal infant dom Henri, en 1558. Il publia la première partie de cette œuvre de grande valeur en $1566{ }^{58}$.

Antonio Galvão, pour sa part, a remis à l'imprimerie son Tratado... dos diversos e desvairados caminhos por onde no tempo passado a pimenta e especiaria veio da India..., en 1563.

A des dates aussi rapprochées, mais déjà éloignées de 50 ans des événements qu'ils racontent, écoutons leur relation respective du voyage de Gaspar Corte-Real, en 1500. Commençons par Damião de Góis, grand humaniste et illustre ami d'Erasme, qui connaissait Luther et Melanchton, et très estimé de l'évêque Paulo Sperato et du cardinal Bembo, de cette ère dorée de la Renaissance. Voyons ce qu'il dit au chapitre LXVI de sa chronique de Dom Manuel, qui a soulevé l'ire de certains de ses contemporains. Toujours ce honteux sentiment d'envie, qui croît en proportion inverse de la grandeur du pays où il se produit, le faisait se consumer sans cesse davantage.

Gaspar Corte-Real

"armou hûa nao com ha qual bem equipada de gente, \& de tudo o mais necessario, partio do porto de Lisboa no começo do verão do anno de mil \& quinhētos. Nesta viagem descobrio, pera quella banda do Norte, hua terra que por ser muito fresca, \& de grândes aruoredos, quomo ho sam todas has que jazê pera aquella bãda, lhes pos nome terra verde. Ha gente da qual he muito barbara, \& agreste, quasi do modo dos da terra da Sancta Cruz, se não q. sam aluos, \& tã cortidos do frio que ha aluura se lhes perde cõ ha idade \& ficã quomo bacos. Sam de corpo meãos, muito ligeiros, \& grandes frecheiros, seruem-se de paos tostados em lugar de azagaias, cõ que ferem de arremesso quomo se fossem forrados de aço fino: vestē se de pelles de alimarias, de que na terra ha muitas. Viuem em cauernas de rocha, \& choupanas, nam tem lei, crem muito em agouros: guardã matrimonio, \& sam muito çiosos de suas molheres, nas quaes cousas se 
pareçem com hos Lapos, que tambem viuẽ de baixo do Norte, de lxx atte lxxxv graos sugeitos ahos Reis de Noroega, \& Sueçia, ahos quaes pagam tributo, ficando sempre em sua gentilidade, por falta de doctrina da qual tyrania no liuro que cõpus da fé, costumes, \& religiam dos Ethiopios, Abexis em lingoa latina, dedicado aho Papa Paulo Terçeiro, no fim delle fiz hua deploraçam em que tratto per extenso dõde este tamanho mal proçede. $\mathrm{E}$ tornãdo a Gaspar Corte Real, depois que descobrio esta terra, \& costeou hũa boa parte della se tornou ao Regno..."

(“... arma une caravelle d'un bon équipage et de tout ce qui lui était nécessaire pour le voyage et partit du port de Lisbonne au début de l'été de 1500. Au cours de ce voyage, il découvrit, au Nord, une terre où la température était très fraîche et où il y avait de grands bosquets, comme dans toute cette contrée; il lui donna le nom de terre verte. Les indigènes de cette contrée étaient barbares et rudes et ressemblaient à ceux de Santa Cruz, sauf qu'ils étaient plus blancs et semblaient rapetissés par le froid, et à mesure qu'ils vieillissent, ils deviennent gris. Ils sont plutôt petits, très agiles et bons archers: en guise de flèches, ils se servent de bâtons dont ils aiguisent la pointe aussi bien que s'il s'agissait de pointe de fer et ils s'en servent comme armes. Ils sont vêtus de peaux de bêtes, très nombreuses dans cette région. Ils vivent dans des cavernes et des huttes. Ils n'ont pas de lois et ils croient beaucoup aux augures. Ils respectent le mariage et sont très jaloux de leurs femmes; dans ce sens, ils ressemblent beaucoup aux Lapons, qui vivent également au nord, entre le $70^{\mathrm{e}}$ et le $85^{\mathrm{e}}$ degrés de latitude, et sont sujets des rois de Norvège et de Suède auxquels ils paient tribut, mais qui demeurent toujours sauvages à cause de leur manque de doctrine ... Corte-Real fit demi-tour après avoir découvert cette terre; il suivit la côte pendant assez longtemps puis revint au Portugal).

De son côté, Galvão s'exprime ainsi :

"Neste mesmo anno de quinhentos diz q pedio Gaspar corte real licença a el Rey dom Manoel pera yr descobrir a terra Noua. Partio da ilha 
terceira com dous navios armados a sua custa, foy áquella clima que está debaixo do norte em cincoênta graos daltura. He terra que se agora chama de seu nome, tornou a saluamento à cidade de Lisboa..." ("En cette année 1500, on dit que Gaspar Corte Real demanda autorisation au roi dom Manuel pour aller à la découverte de la terre Neuve. Il partit de l'île Terceira avec deux navires gréés à ses frais, et il se dirigea vers cette région nordique qui se trouve à cinquante degrés de latitude. Cette terre porte maintenant son nom. Il revint sain et sauf à Lisbonne").

D'après le premier, Gaspar s'embarqua à Lisbonne, au début de l'été de 1500. Mais d'après Galvão, le départ de l'expédition aurait plutôt eu lieu à l'île Terceira, alors que le navigateur partait avec deux caravelles, gréées à ses frais. Pour celui-ci, la terre rencontrée serait Terre-Neuve, et il affirme que le nom de Corte Real la désigna ainsi par la suite. Or, il ne semble pas y avoir de doute qu'il s'agissait de la partie orientale du Groenland actuel ${ }^{59}$. Gaspar aurait même doublé le cap Farewell, en suivant la côte sud-ouest. Près de la baie de North Ström, à $67^{\circ} 30^{\prime}$, l'explorateur fut de nouveau arrêté par des montagnes de glace. Il repartit alors pour Lisbonne où il arriva à l'automne de cette année-là.

C'était son premier voyage après les lettres patentes de 1500 , déjà mentionnées, qui lui assuraient la possession de ses découvertes.

Le voyage suivant devait se terminer tragiquement par la disparition de la caravelle qui transportait cet "homem auentureiro, esforçado, \& desejoso de ganhar honra" ("homme aventurier, courageux et désireux de remporter des honneurs") comme disait Damião de Góis.

Tout ce que nous savons de cette nouvelle exploration du nord-ouest atlantique nous vient surtout des descriptions de Pasqualigo et de Cantino, jointes à la carte qui porte le nom de ce dernier. Henry Harrisse en a fait une étude très fouillée qui nous a servi très souvent de référence. 
Dans l'édition de langue française de notre travail "La Découverte de Terre-Neuve", nous appelons Alberto Cantino "l'espion italien". Nous aurions pu tout aussi bien l'appeler "l'observateur étranger"! Voyons donc ce que dit le célèbre commentateur des voyages de Corte-Real ${ }^{60}$ :

... Nous ne savons en quelle qualité il habitait le Portugal. Bien qu'il intitule son noble correspondant - Dignissimo ac Domino Singularissimo, rien ne prouve qu'Alberto Cantino fut agent diplomatique ou orateur, ainsi qu'on disait alors, du duc de Ferrare. Nous avons vainement fait chercher son nom dans les listes des fonctionnaires de la maison d'Este. Il n'est pas même certain qu'il fut Ferrarais. Ce n'était peut-être qu'un de ces négociants italiens, établis dans les ports de la péninsule, comme Lorenzo Girardi, Simone del Verde, Berardi, Bartolomeo Marchioni et son facteur Giovanni da Empoli, Benvenuto Benvenuti, Francesco Lotti, etc., etc., qui, sans cesse à l'affût de nouvelles des pays lointains s'empressaient de les communiquer à leurs parents et amis, quand ils ne correspondaient pas directement avec leurs princes ou les gens de la cour.

Quoi qu'il en soit, nous devons à cet homme une carte géographique précieuse qui, ironie du sort, l'a immortalisé; elle est connue aujourd'hui sous le nom de planisphère de Cantino. Grâce à elle, nous pouvons suivre, d'une certaine façon, les voyages de Gaspar Corte-Real, et connaître l'état de nos découvertes à cette époque. Le présumé agent du duc Hercule d'Este, bien payé par ce dernier (douze ducats d'or, admit-il lui-même ${ }^{61}$ ), obtint d'un certain cartographe portugais, traître à son prince, des révélations pourtant bien gardées.

Ce Pietro Pasqualigo, au contraire, se trouvait à Lisbonne à titre d'ambassadeur, ou d'orateur, terme employé alors, par la Seigneurie de Venise. Il était de ces nombreux agents officiels de la cité de Lagune qui inaugurèrent un style et une présence en pays étranger, et que nous appellerons plus tard la diplomatie. Cette diplomatie s'est transmise jusqu'à nos jours sous une forme qui, malgré les apparences, n'a pas subi de 
modification dans sa structure. Par conséquent, Pasqualigo devait s'en tenir uniquement à cette formule, née avec la diplomatie permanente, soit l'observation. Chazelle ${ }^{62}$ écrivait récemment: “...En installant, au contraire, à demeure près de souverains étrangers un observateur à sa solde, l'État qui l'y dépêche montre l'intérêt qu'il attache à suivre en permanence l'évolution de ces pays et à en tenir régulièrement compte dans sa politique extérieure: intérêt d'autant plus vif que les cités italiennes où s'instaura cette pratique étaient d'une extrême faiblesse contre les périls du dehors, et ne pouvaient la compenser que par une connaissance précise, constamment tenue à jour, des ressources, des lacunes et plus encore des intentions de leurs adversaires éventuels."

Or, les routes de l'Inde que nous cherchions à découvrir soit en contournant l'Afrique ou soit en tentant de trouver un passage par l'Occident, menaçaient de déplacer le centre du commerce oriental en Europe: de Venise et de Gênes vers Lisbonne, ce qui se produisit par la suite ${ }^{63}$. L'on voit à quel point étaient importants, surtout pour la péninsule italienne, tous les renseignements recueillis dans ce petit pays de l'extrémité occidentale de l'Europe qui se lançait, comme un géant, dans la grande aventure des découvertes. Nous avons finalement atteint la terre des épices, repoussant les Musulmans par surprise sur leur arrière-garde, et avons étendu notre empire jusqu'à Malacca, avec Alphonse d'Albuquerque, et ensuite, embrassant d'une étreinte spirituelle la Chine intérieure, quand nous nous sommes établis à Macau. Lisbonne était alors devenue le centre d'un grand empire commercial. Mais cette grandeur a passé comme un éclair, par la faute du Portugal. En expulsant les Juifs (1496), d'une part nous nous privions de l'unique classe de notre société qui tenait alors la clé de notre économie intérieure; et d'autre part, nous nous aliénions la plus puissante maçonnerie internationale connue: celle des Hébreux, qui orientait la finance et le commerce européens ${ }^{64}$. Un grand poète portugais de cette époque, Sá de Miranda, s'est exclamé, étreint par l'émotion: "como eu vi correr pardaus por Cabeceiras de Basto !" ("comme j'ai vu courir la monnaie des Indes 
à Cabeceiras de Basto !"). En vérité, l'or de l'Orient a brillé un jour jusque sur les plus petits villages portugais !

En comparant les diverses sources d'information dont nous disposons, nous ne pouvons fixer de façon précise la date du départ de Gaspar Corte-Real pour cette nouvelle expédition. Pour Damião de Góis, ce serait le 15 mai 1501, date qu'admet Biggar; pour sa part, Cantino ${ }^{65}$ déclare, au début de sa lettre du 17 octobre 1501, que "Gia son nove mesi passati che questo Serenissimo Re mando alle parte de Tramontana due legni ben armati solum per cerchare se possibil fusse che a quella parte vi si possesse ritrovare terre on insule alcune," et que le 11 de ce même mois, une des caravelles était revenue. De son côté, Pasqualigo affirme, dans sa lettre du 18 octobre 1501: "A di 9 dil presente arivo qui una di due caravelle quale l'anno passato la Maiestà del dito re mando a discoprir terra verso la parte de tramontana..." Il est possible que ce dernier ait confondu ce voyage avec celui de 1500 en ce qui a trait aux dates de départ des navires. De toute façon, il est curieux de constater comment deux personnes, écrivant presque en même temps au sujet d'événements qui viennent de se dérouler, en relatent les faits de façon aussi différente !

En confrontant les dires de Cantino avec le planisphère qui porte son nom, on ne peut reconstituer, comme le fait Biggar, que de façon approximative ce voyage de Gaspar Corte-Real. Ce dernier, pour ses contemporains comme pour l'éditeur portugais du livre de Marco Polo, Valentim Fernandes, aurait découvert la pointe occidentale des provinces orientales de "Tenduch, Mangy, Tanguth": "o principio das quaes segundo o meu pequeno saber achou o muy honrado fidalguo Gaspar Corte Real" 66 ("dont le très honoré seigneur Gaspar Corte Real, selon mon humble opinion, aurait découvert le commencement").

Cantino aurait entendu le récit du voyage d'un membre de l'équipage au retour de la caravelle, et il aurait vu les indigènes emmenés à Lisbonne; ce furent les premiers habitants du continent américain à mettre le pied sur le sol européen. L'informateur du duc de Ferrare raconta que cette expédition partit 
de Lisbonne (Biggar croit qu'il s'agit plutôt de l'île Terceira) et, durant "quatre mois", navigua vers le Nord sans rencontrer signe de vie. Au début du "cinquième mois", des montagnes de glaces flottantes l'obligèrent à changer de route, dans la direction ouest et nord-ouest. Edgar Prestage précise qu'il y eut confusion manifeste des mois et des semaines ${ }^{67}$.

Mais "trois mois" (semaines) se passèrent durant lesquels ils jouirent d'un temps favorable à la navigation, et au premier jour du quatrième mois (semaine), ils aperçurent la terre ferme: "Et nel primo giorno del quarto mese heberno vista ... d'un grandissimo paese, alquale con grandissima allegreza se acostarno, et correndo molti et grandi fiumi dolci per quella regione al mare, per uno de epsi forsi una legha fra terra intrarno, et in quella dismontati trovarno copia de suavissime et diversi fructi, et albori et pini de si smisurata alteza et grosseza che serebbeno troppo per arboro de la piu gran nave che vadi in mare. Ivi non nasce biada d'alcuna sorte, ma gli homini do quel paese dicono non vivere se non de pescasone et caza de animali, de liquali el paese abonda, ciocè cervi grandissimi, vestiti de longissimo pelo, le pelle de liquali usano per veste, ne fanno case et barche; et cusi lupi, volpe, tigri et zebellini. Affermano esservi, che mi pare miraculo, tanti falcuni peregrini, quante passare sono nel nostro paese, et io ne ho veduti, et sono belletissimi..."

C'était un véritable Shan-gri-la de couleurs merveilleuses, après les aspérités glacées!

D'après Biggar, une fois l'expédition arrivée à la pointe du Groenland, le cap Farewell d'aujourd'hui, elle se serait dirigée vers le nouveau continent et l'aurait touché à la hauteur du Labrador actuel; il est même possible que ce soit près du cap Mugford. Le parcours aurait donc été à peu près le même que celui du voyage précédent, sauf que, cette fois, Corte-Real ne s'est pas éloigné du continent; il longea la côte en direction du sud. C'est ainsi qu'il serait arrivé à l'île de Terre-Neuve. Les cartes géographiques de l'époque sont couvertes, à cet endroit, de noms bien portugais, dont certains sont d'ailleurs restés, 
confirmant le passage de celui qui s'est perdu ensuite pour toujours, probablement dans les mers environnantes.

Morison, malgré le scepticisme qu'il affiche dans tout ce qu'il dit au sujet de notre découverte dans toutes les directions du continent américain, est tout de même forcé d'admettre: "And, as there is no evidence of John Cabot having coasted further north than his landfall at Cape Breton, Gaspar CorteReal should be regarded as the real discoverer of Newfoundland". ${ }^{68}$

Cantino et Pasqualigo décrivent avec force détails les Indiens emmenés au pays; curiosité bien naturelle envers cette nouvelle race d'êtres humains qui montraient beaucoup de traits de ressemblance avec ceux de l'Extrême-Orient dont l'Européen avait déjà entendu la description. Tout portait à confondre le Nouveau-Monde avec les très vieilles terres des épices où l'on trouvait ces pierres précieuses que Polo avait rapportées dans ses bagages et dans ses souvenirs émerveillés.

Le voyage de retour des deux caravelles arrivées à Lisbonne, l'une le 9 et l'autre le 11 octobre, avait duré à peine un mois. Il manquait celle de Gaspar Corte-Real, qui était resté pour explorer cette région parce qu'il croyait (c'est ce que nos marins rapportèrent à l'Orateur de Venise) ce continent relié aux terres aperçues lors de son expédition précédente. Il voulait également chercher si, entre ce nouveau monde et les Antilles, il existait également un lien et aussi avec la "terre des perroquets", comme nous commencions à appeler le futur Brésil: “...Scop (er) to una terra noua la chiamão d(e) li Papaga ..." Ainsi le décrivait Lorenzo Cretico, autre ambassadeur de la Lagune à Lisbonne, au retour de Pedro Alvares Cabral ${ }^{69}$. Telle était l'impression causée par ces oiseaux colorés qu'on y avait rencontrés et que bientôt le cartographe de Cantino dessinait pour représenter la nouvelle découverte portugaise.

Pasqualigo s'exprimait ainsi: "Credeno questi di la caravella la soprascrita terra esser terra ferma, et, conjungerse con altra terra, laqual l'anno passato soto la tramontana fu disco- 
perta da l'altre caravele de questa Maiestà. Licet non potesseno arrivar a quella per esser el mar li agiazato con grandissima quantità di neve in modo che monti. Qual terra etiam credeno conjungerse con le Andilie, che furono discoperte per li Reali di Spagna, et con la terra dei papaga, noviter trovata per le nave di questo re che andorono in Calicut. El creder questo se moveno, prima, perche havendo corsa la costa de dita terra per spazio de 600 et piu miglia, non hanno trovato fin alguno; poi perche diceno haver trovate molte fiumare grossissime, che li meteno in mare. Expetasse di zorno in zorno l'altra caravella capetania, da laqual distinctamente se intendera la qualità et condition ch'è la sopradita terra, per esser andata piu avanti scorendo per quella costa, per discoprir quanto piu potra di quella."

Mais Gaspar Corte-Real ne revint jamais plus!

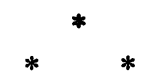

Henry Percival Biggar ${ }^{70}$, qui fut un grand chercheur canadien dans le domaine des origines de son pays, fut également le premier qui, sur le continent américain, donna au Portugal, quoique timidement, sa véritable place de pionnier des mers de l'Atlantique-Nord, au cours de la redécouverte du NouveauMonde. Il applique à la toponymie gaspariana de Terre-Neuve certaines observations basées sur les cartes et les quelques documents connus, déjà mentionnés ici. Il est intéressant de noter la façon dont il suit l'itinéraire du dernier voyage de Gaspar Corte-Real en accolant les noms qui commencent à baptiser les caps, les baies et les promontoires ${ }^{71}$ :

"Continuant sa route vers le sud, il (Gaspar Corte Real) prit le détroit de Belle-Isle pour une baie et supposa que la Côte orientale de Terre Neuve était la continuation de la côte principale déjà explorée au nord. Belle-Isle fut appelée île Friar Lewis, mais le cap Bonavista a conservé le nom de cabo de Boa Vista ou cap Fairview qui lui fut donné par Corte Real. La baie Trinity fut appelée baie Saint-Irénée, nom d'un saint portugais bien connu. Plus loin au sud, l'île Baccalieu et la baie 
Conception ont conservé, toutes les deux, les noms qui leur furent donnés par Gaspar. Ferryland reçut le nom de Farelhão ou promontoire rocheux, et Fermeuse fut appelée rio Fermoso ou belle rivière. Le cap Race doit son nom à son apparence stérile $(c a b o$ Razo) ou à sa ressemblance à un cap du même nom situé à l'embouchure du Tagus.

Après avoir contourné ce promontoire, Corte Real longea les côtes du sud de Terre Neuve jusqu'à la baie Placentia qu'il nomma la grande Baie. Quant à la baie Sainte Marie située entre cette dernière et le cap Race, il semble que l'explorateur lui donna le nom de havre de la Croix, si l'on tient compte de la fête qui fut célébrée le 14 septembre.

Ce fut vers cette époque que dans l'un des ports sur le côté ouest de la baie Placentia, Corte Real se sépara de deux de ses vaisseaux qui prirent la route de Lisbonne."

Milton Alexander Buchanan ${ }^{72}$, qui fut professeur à l'université de Toronto et un grand hispaniste, nous a laissé une intéressante étude, intitulée "Notes on portuguese place-names in North-Eastern America" 73. Ce fut peut-être la première tentative sérieuse qui souleva le voile sur la toponymie terreneuvienne, invitant à une étude approfondie sur l'origine et l'évolution des noms géographiques de cette île: "Something of the history of Newfoundland needs to be recalled to understand the mutations in form and meaning which some place-names have undergone". Et un peu plus loin (p. 102): “Examples used incidentally in this paper have shown how tenacious of survival Portuguese place-names have been in this region much more so than the names applied by Cartier and Champlain in adjoining territories... The reason for the survival of Portuguese names seems to be that they designated bays, capes and islands which were important marks for navigation and that they were in uninterrupted use by succeeding generations, even though of different race and language. They distorted them but could not dispense with them or destroy them."

La présence portugaise dans cette région, malgré l'oubli où a sombré l'œuvre si extraordinaire de nos navigateurs sur 
la côte nord de ce continent, a résisté aux rigueurs du temps et des hommes. On peut défendre toutes les légendes sur la redécouverte de l'Amérique, mais la justice de l'histoire finit par triompher, tôt ou tard, même si, pour y arriver, il faut lutter contre le manque de documents et de cartes marines. Demain, sur cette terre qui portait le nom de Corte-Real, la statue de Gaspar qui va s'y dresser sera en même temps une réparation envers cet illustre navigateur que le temps avait laissé presque oublié, mais aussi un hommage au vieux Portugal découvreur et créateur de nouvelles nations, de mondes nouveaux où la parole de l'Évangile fut répandue, sans discrimination de races ou de couleur, aussi bien sur son territoire métropolitain qu'outre-mer.

(à suivre)

EdUARdo BraZ̃̃o,

Ambassadeur du Portugal au Canada.

1 Op. cit., 40; du même auteur, Christophe Colomb, son origine, sa vie, ses voyages et ses descendants, chap. "Les Précurseurs"; il est curieux de constater comme l'œuvre du Portugal fut longtemps méconnue dans cette région. Voir, par exemple, le livre de Thomas Rundall, publié par "The Hakluyt Society" en 1849, Narratives of voyages towards the North-West, in search of a passage to Cathay and India, 1496 to 1631, with selections from the early records of the Honourable the East India Company and from mss. in the British Museum, publié récemment par Burt Franklin, de New York. Cet ouvrage, très répandu à l'époque, commence avec Sir Hugh Willoughby et William Adams, vient ensuite John Cabot, son fils Sébastien, en passant par Sir Martin Frobisher, John Davis, George Waymouth, John Knight, Henry Hudson, Sir Thomas Button, James Hall, Bylot et Baffin, Hawkridge, Luke Fox, James. Comme on le voit, on ne mentionne aucun Portugais, et pourtant, ils furent les premiers et sans doute les plus importants. Sur les voyages de Frobisher, voir "Three voyages of Martin Frobisher on search of a passage to Cathaia and India by the North West, A.D. $1576-78$, reprinted from the first edition of Hakluyt voyages, with selections from manuscript documents in the British Museum and State paper office by Rear-Admiral, Richard Collinson, C.B. nouvelle éd. par Burt Franklin, de New York.

2 Samuel Eliot Morison, Portuguese Voyages to America in the Fifteenth Century (Cambridge, Mass., 1940), 21.

3 Edgar Prestage, The Portuguese Pioneers (Londres, 1933), 40. (II existe une traduction portugaise de cette œuvre sous le titre: Descobridores Portugueses, par Francisco Eduardo Baptista, Porto, 1934, 1943) et Morison, op. cit., 12, signale Teive: "a well known Madeiran, a squire of Prince Henry who ordered him in 1452 to build the first sugar mill on that Island"; J. M. Silva Marques, Descobrimentos Portugueses, Suplemento ao tomo $I$ : 343 et 570, tome I: 515-516. 
4 Morison, op. cit., 14: "Corvo and Flores westermost of the group and not visible from any others, were probably discovered by Diogo de Teive in or shortly before 1452; for on January 20, $1453 \mathrm{D}$. Afonso V granted Corvo to this uncle D. Afonso Duke of Bragança, and Flores to Dona Maria de Vilhema mistress of the robes to the Infanta Dona Leonor. Upon the death of Prince Henry in 1460 the proprietorship of the other seven islands was transferred to his nephew and adopted son D. Fernando Duke of Viseu (1433-74). By that time most of the islands had been settled by energetic and enterprising colonists, many of them Flemings, who like the pioneers of New England and the West Indies combined agriculture with trading and fishing."

Voir également Manuel Monteiro Velho Arruda, Colecção de documentos relativos ao descobrimento e povoamento dos Açôres, Ponta Delgada (1932); Jules Mees, Histoire de la découverte des Iles Açores (1901); Damião Peres, História dos Descobrimentos Portugueses, 69 et suiv.; Manuel C. Baptista de Lima, "Deux voyages portugais de découverte dans l'Atlantique occidental", dans le Bulletin des Etudes Portugaises de l'Institut Francais au Portugal, X, fasc. I (Coïmbra, 1945), 42 et suivantes.

5 Jaime Cortesão, op. cit., I : 384 et suiv.; du même auteur, le résumé des cours qu'il donna à l'université de Séville en 1933, sous le titre de "A viagem de Diogo de Teive e Pero Vasquez de la Frontera ao Banco da Terra Nova em 1452", dans Arquivo Histórico da Marinha, I, n 1 (Lisbonne, 1933). De Cortesão également, sur le même sujet, sa collaboration au tome III de Historia de America $y$ de los pueblos americanos, dirigé par Antonio Ballesteros Beretta (Barcelone, Buenos-Aires, 1947), chapitre intitulé "Los Portugueses", 659 et suivantes. Il est intéressant de signaler que le premier titre de cette étude était "Los descubrimientos pre-colombinos de los portugueses". Nous ignorons, et l'auteur ne le dit pas, pourquoi cette modification a été apportée par la suite. Voir dans les "Obras completas de Jaime Cortesão", vol. V: $A$ expansã̃o dos Portugueses no periodo Henriquino, chapitre "A viagem de Diogo de Teive e Pero Vasquez de la Frontera aos bancos da Terra Nova em 1452", p. 187 et suivantes.

6 Le Historie della Vita e dei Fatti di Cristoforo Colombo per $D$. Fernando Colombo, suo figlio (Venise, 1571), chap. IX, traduction italienne de l'original perdu.

7 "Historia de las Indias par Fr. Bartolomé de Las Casas, publicada ahora por vez primeira, conforme à los originales del autor, que se custodian en la Biblioteca de la Academia de la Historia y en la Nacional de esta corte", dans la "Coleccion de Documentos inéditos para la Historia de España por el Marqués de la Fuensanta del Valle y José Sancho Rayon", tomes LXII, LXIII, LXIV, LXV et LXVI (Madrid, 1875 et 1876). C'est cette édition que nous utilisons et citons.

8 Dans Las Casas (chap. XIII: 100 du tome LXII des Documentos Inéditos...): "Algunos salieron de Portugal á buscar esta misma, que por comun vocablo, la llamaban Antilla, entre los cuales salió uno que se decia Diego Detiene, cuyo piloto, que se llamó Pedro de Velasco, vecino de Palos ...."

9 Comme on l'a vu dans les notes antérieures, Las Casas a évité cette erreur, car Palos est en Andalousie (Espagne).

10 Las Casas (cit. 100): “...y andurieron 150 leguas por el viento lebechio, que es el viento Norueste..."

11 Las Casas (cit. 100) : "fueron por el Nordeste..."

12 Las Casas (cit. 100), “... se les quebada el Cabo de Clara, que es en Ibernia, hácia el Leste..." 
13 Las Casas (cit. 100): “... lo cual no prosiguieron yendo para descubrirla (les Sete Cidades), porque era ya por Agosto y temieron el invierno..."

${ }^{14}$ Las Casas (cit. 100) : “... Esto diz que fué cuarenta años ántes que el Cristóbal Colon descubriese nuestras Indias ..."

15 Las Casas (101): “...y esta creyeron los que alli iban que debia de ser la que quiso descubrir un Hernan Dolinos, como luégo se dirá..." 16 Jaime Cortesão, Os Descobrimentos Portugueses, I: 386.

17 Colon y Pinzon (1883); J. Cortesão écrit (op. cit., I: 388) : “... (dans) les témoignages desdits procès qui se rapportent à la période de 1532 à $1535 \ldots$ on mentionne un certain Pero Vasquez de la Fronteira, pilote de Palos, qui informa Colomb que, étant au service du roi de Portugal, il avait navigué vers l'Occident, et il lui affirmait avec certitude l'existence d'une terre dans cette direction."

18 Duarte Leite, op. cit., I: 355 , présente des objections à l'identification des pilotes: "Le premier doute que suscite la comparaison du texte de F. Colomb avec les dépositions vient de la coïncidence ou la différence des voyages de Teive et de Pero Vasques. J. Cortesão opte pour la coïncidence, en s'appuyant sur l'identité de ses pilotes: Pero de Velasco serait la traduction, en espagnol, du nom latin Petrus Velascus ou Velasci, car la note de Colomb, où il donnait le texte à son fils, était rédigée en latin et elle correspond à Pero Vasques. On peut soulever deux objections à cette identification: tout d'abord, le fait que la note originale ait été écrite en latin est contestable, puisque les arguments présentés ne permettent pas de le supposer; et ensuite, l'un des pilotes était né à Palos de Moguer et l'autre à Fronteira; c'est une différence importante et oubliée. En plus de cette divergence, deux circonstances concourent à distinguer les deux voyages: dans celui de Pero Vasques, les témoins ne parlent pas de la découverte de l'île Flores ni, surtout, de la navigation vers le nord-ouest, point essentiel de la thèse discutée. Il convient donc d'admettre que le voyage de Pero Vasques s'est fait en direction de la terre découverte plus tard par Colomb, la seule à laquelle font allusion les pilotes et les témoins. Il est postérieur au voyage de Teive, rapporté par F. Colomb; et l'Infant qui l'ordonna peut bien être dom Ferdinand, qui favorisa les explorations de l'Atlantique par le sud-ouest, dont l'une atteignit la mer des Sargasses que les Portugais connaissaient déjà."

19 Jaime Cortesão, op. cit., vol. I: 389.

20 Drumond, Anais da llha Terceira, tome I, Angra do Heroismo, (1850), 26; Manuel de Macêdo, Nobiliário, mss. 402 dans la "Coleç̧ão Pombalina" (Biblioteca Nac. de Lisboa), fol. 220.

21 Jaime Cortesão, op. cit., I: 386 et suivantes.

22 Morison utilise tous les arguments possibles pour éloigner les navigateurs portugais de la côte américaine. C'est ainsi qu'il répond au point de vue de Jaime Cortesão, déjà mentionné: "Having altered their course a mere ninety degrees to bring them to the New World instead of the Old Ireland, Cortesão declares that only one spot in the North Atlantic would satisfy the description of their furthest north: "the Newfoundland bank, near the Avalon peninsula." Here the winds would be west, and the water smooth, because under the lee of Newfoundland" (cit. de Geogr. Journ., LXXXIX: 32 et suiv.).

To answer to this identification it may be observed that the protection of a weather shore does not extend further than it can be seen, nor, in general, nearly so far; and Newfoundland is forty-five nautical miles distant from the nearest edge of the Grand Bank. Those waters, according 
to my memory of some six or eight summer crossings, are uncommonly rough. Dr. Cortesão's assurance to the contrary so shock my confidence on this point, that in April, 1938, I consulted several masters of the Lisbon banker fleet, then ready to sail for their summer's fishing off Newfoundland; and was relieved to have my own nautical memories indicated by these practical seamen. As one of them said, "Naturally it's rougher on the Banks than outside, if the wind is of equal force, because the water is shallow. It's always rougher in shoal water, as you can see for yourself on the Bar of Lisbon." And another passed some remarks very uncomplimentary to armchair admirals and library navigators" (op. cit., p. 23 et suiv.).

23 Morison, op. cit., 29.

24 Frank Debenham, Discovery and Exploration, an atlas-history of man's journeys into the unknown, introduction by Edward Shackleton. Voir aussi le récent ouvrage de Samuel Eliot Morison, The Oxford History of the American people (New York, 1965), 22.

25 Jaime Cortesão, op. cit., I: 384.

26 Bartolomeu de Las Casas écrit, p. 97 du vol. cité, chap. XIII: "Dico, pues, Cristóbal Colon entre otras cosas que puso en sus libros por escrito, que hablando con hombres de la mar, personas diversas que navegaban las mares de Occidente, mayormente á las islas de los Azores y de la Madera, entre otras, le dijo un piloto del rey de Portugal, que se llamaba Martin Vicente, que ballándose una vez 450 leguas al Poniente del Cabo de San Vicente, vido e cogió en el navio, en el mar, un pedazo de madero labrado por artificio, y, á lo que juzgaba, no con hierro: de lo cual y por haber muchos dias ventado vientos Ponientes, inaginaba que aquel palo venia de alguna isla ó islas que hácia el Poniente hobiase. Tambien otro que se nombró Pero Correa, concuño del misno Cristóbal Colon, casado con la hermana de su mujer, la certificó que en la isla del Puerto Sancto habia visto otro madero venido con los mismos vientos y labrado de la misma forma, é que tambien habia visto cañas muy gruesas, que en un canuto dellas pudioran caber tres azumbres de agua ó de vino; y esto mismo dice Cristóbal Colon que oyó afirmar al Rey de Portugal, hablando con el en estas materias, y que el Rey se las mandó mostrar. El cual tuvo por cierto (digo el Cristóbal Colon) ser las dichas cañas de algunas islas ó isla que no estaba muy léjos, ó traidas de la India con el impetu del viento y de la mar, pues en todas nuestras partes de la Europa no las habia, ó no se sabia que las hubiese semejantes. Ayudábale á esta creencia que Ptolomeo, en el lib. I, cap 27 de su Cosmographia, dice que en la India so hallaban de aquellas cañas. Item, por algunos de los vecinos de las islas de los Azores, era certificado Cristóbal Colon, que ventanto vientos recios Ponientes y Noruestes, traia la mar algunos pinos y los echaba en aquellas islas, en la costa, en especial en la isla Graciosa y en la del Fayal, no habiendo por parte alguna de aquellas islas donde se hallase pino. Otros le dijeron que en la isla de las Flores, que es una de las Azores, habia echado la mar dos cuerpos de hombres muertos, que parecia tener las caras muy anchas y de otro gesteo que tienen los cristianos; otra vez, diz, que en el Cabo de la Verga que es en (está en blanco en el original), y por aquella comarca, se vieron almadias ó canoas con casa movediza, las cuales por ventura, pasando de una isla á otra, ó de un lugar á otro, la fuerza de los vientos y mar las echó donde, no pudiendo tornar los quo las traian, perecieron, y ellas, como nunca jamás se hunden, vinieron á parar por tiempo á los Azores."

Voir Henrique Braz, op. cit., 13, citant Antonio de Herrera; Edgar Prestage, op. cit., dans l'édition portugaise de 1943, 271. 
27 Henry Harrisse, op. cit., 41 et suiv.; Morison, op. cit., chapitre intitulé: "Efforts at Western Discovery, 1462-1475", p. 29 et suivantes.

28 Manuel Monteiro Velho Arruda, Colecção de documentos relativos ao descobrimento dos Açôres, Ponta Delgada, 1932, 147 et suiv.; José Ramos Coelho, Alguns documentos do Archivo Nacional da Torre do Tombo àcerca das navegações e conquistas portuguesas, (Lisbonne, 1892), 28 et suiv.; Morison, op. cit., 31.

29 Duarte Leite, op. cit., I: 31.

30 Velho Arruda, op. cit., 150; Alguns documentos..., 32.

31 Jaime Cortesão, op. cit., I: 411; Edgar Prestage, op. cit., 229, eroit, au contraire, qu'il s'agissait de l'une des Antilles: “...possible one of the Antilles" et Morison commente, plus justement cette fois (op. cit.,, p. 31, note 49), que cela "is incompatible with the direction given, with the prevailing winds, and with the known route of Portuguese ships returning home from that part of Africa."

32 Morison, op. cit., 32; Velho Arruda, op. cit., 157.

33 Damião Peres, História dos Descobrimentos Portugueses, 165; Jaime Cortesão cite la page 412 du vol. I de son ouvrage cité.

34 A. J. da Silva D'Azevedo, Américas, um corolário de Sagres, Lisbonne, 1964, 113.

35 Jaime Cortesão, op. cit., II : 147 et suivantes.

36 Damião Peres, História dos Descobrimentos Portugueses, édition du centenaire d'Henri, 74; Jaime Cortesão, Introdução à História das Bandeiras, I: 180, écrit: "A ce concept de l'existence possible de terres entre l'Europe occidentale et l'Orient asiatique, les Portugais ont donné la forme mythique d'Antilha, vaste île ou continent qui apparaît sur nombre de cartes depuis le début du $X^{e}$ siècle, dans l'Atlantique ou dans le Pacifique. Fernando Colomb, dans l'œuvre qu'il a consacrée à la mémoire de son père, et lorsqu'il se reporte aux notes de celui-ci, attribuait aux Portugais la désignation d'Antilles. Plusieurs auteurs de cartes l'ont identifiée avec l'île légendaire des Sept Cités, également une création portugaise, comme on le retrouve dans la célèbre lettre de Toscanelli au chanoine Fernand Martins, en 1474. Sur d'autres cartes de la première moitié du XVIe siècle, les noms, soit d'Antilha, soit de Sept Cités, apparaissent pour désigner les régions d'Amérique mal connues, ou des terres du Pacifique totalement inconnues. Le mythe continuait de conserver sa valeur d'hypothèse cosmographique, préfiguration d'un monde plus vaste en harmonie avec une expérience plus considérable et la culture nautique des Portugais.

C'est sur cet ensemble de connaissances et de mythes qui peuvent avoir valeur d'hypothèses scientifiques que se fondèrent les conseillers de Jean II pour s'objecter au projet de Colomb. Mais les efforts soutenus du monarque portugais au cours des négociations de Tordesilhas pour obtenir le déplacement du méridien, enfin couronnés de succès, ne peuvent s'expliquer par de simples hypothèses ou par des idées mythiques.

A ce sujet, Colomb, nous ne devons pas l'oublier, avait une opinion bien précise. Elle est même très ferme. A compter du 4 juillet 1498, durant son troisième voyage, de Santiago du Cap Vert vers le sud-ouest, Colomb, transcrit par Las Casas, écrivait: "Torna el Almirante a decir que quiere ir al Austro ... quiere ver qual era la intencion de el rey $D$. Juan de Portugal que decia que al Austro habia tierra firme; y por esto dice que tuvo diferencias con los Reys de Castilla, y en fin, dice que se concluyó que el Rey de Portugal hobiese 370 léguas ...", mais à l'ouest de l'archipel. Et il ajoute: "tenia el dicho rey por cierto que dentro de sus 
limites habia de hallar cosas y tierras famosas ... y que el rey D. Juan tenia gran inclinacion de enviar a descobrir al sudoeste..." Et la conviction de Colomb était tellement forte que l'avance du méridien a été justifiée pour ces raisons que "mandó gobernar por la via de sudoeste ... en cual camino veria la opinion del rey D. Juan susodicha (chap. CXXX: 225 et CXXXII: 226, de l'œuvre citée de Las Casas, édition déjà mentionnée).

En admettant, comme nous le faisons, en nous appuyant aussi sur d'autres témoignages, que Jean II connaissait l'existence de terres au sudouest de l'archipel du Cap Vert, ces efforts prennent un sens complet à la lumière de la géopolitique; le Portugal, par le traité de Tordesilhas, garantit la possession de la véritable route de l'Inde et des deux rivages, africain et américain, de l'océan que cette route traverse. Il assurait la défense de sa grande route commerciale et commençait le partage d'un monde à explorer.

De son côté, Morison (op. cit., 16) écrit: "South of St. Brendan chain, on the Pareto map (1455), are the authentic Madeira and Canary groups. West of St. Brendans, in about longitude $25^{\circ} \mathrm{W}$ and between latitude $34^{\circ}$ et $40^{\circ} \mathrm{N}$ (judging from the scale of the Iberian Peninsula) is the Island of Antilia. This name, from which the Antiles derive theirs, probably originated in a series of cartographer' blunders from Getulia, the classical name for the northwestern part of Africa. The Pizzigani Map of 1367 contained an inscription in the sea off Portugal: "Here are statues which stand before the shores of Atullia" i.e., Getulia; and later map makers supposed that Atullia was an island, and corrupted the name further to Antilia. The Portuguese, when they first saw Antilia on maps, identified it with the Ilha das Sete Cidades (Island of the Seven Cities) in their own folklore. That island was supposed to have been settled by seven bishops and sundry faithful, who fled from the Peninsula during the barbarian conquest in the eighth century."

Voyons maintenant ce que disaient les anciens. Las Casas écrivait (op. cit., 99 du volume mentionné qui comprend le chapitre XIII) :

"Esta isla do las Siete Ciudades, dicen, segun se suena, los portugueses, que fuè poblada dellos al tiempo que se perdiồ España reinando el rey $D$. Rodrigo; y dicen que por huir de aquella persecucion se embarcaron siete Obispos y mucha gente, y con sus navios fueron á aportar á la dicha isla, donde cada uno hizo su pueblo, y porque la gente no pensase tornar, pusieron fuego ás los navios, y dicese que en tiempo del Infante D. Enrique de Portugal, con tormenta, corriò un navio que habia salido del puerto de Portugal y no paró hasta dar en ella, $y$, saltando en tierra, los de la isla los elevaron à la iglesia por ver si eran Cristianos y hacian las cerimonias romanas, y visto que lo eran, rogáronles que estuviesen alli hasta que viniese su señor que estaba de alli apartado, por los marineros temiendo nos les quemasen el navio y los detuviesen alli, sospechando que no querian ser sabidos de nadie, volviéronse á Portugal muy alegres esperando recibir mercedes del Infante; à los cuales diz que maltrató e mandó que volviesen, pero el maestre y ellos no lo osaron hacer, por cuya causa, del reino salidos, nunca más á él volvieron: dicen más, que los gromettes cogieron cierta tierra ó arena para su fogon, y que hallaron que mucha parte della era oro..."

Antonio Calvão, dans son Tratado... déjà cité, (réédition citée de Burth Franklin, 72) écrit:

"Contam mais que nestes meyo tempo vindo hũa nao de Portugueses pelo estreito de Gibraltar fora, lhe dera tal tromẽta, q' correra a loeste muito mais do q' quisera, \& forã ter a hũa ilha em q' auia sete cidades \& falauã a nossa lingoa \& preguntarã se tinham os mouros ainda occupada 
Espanha dõde fogirã pola perda del rey dõ Rodrigo. O contra mestre da nao diz q' trouze hũa pouca d'area \& q' a vendera a hũ ouriues em Lixboa de q' tirara boa cãtidade douro: sabendo isto ho Infante dõ Pedro que ainda gouernaua, diz q' ho mãdou escreuer na casa do tõbo. $\mathbf{E}$ algũs querem q' estas terras \& ilhas q' os Portugueses tocarã, sejam aquelas que se agora chamã as Antilhas \& noua Espanha, \& alegam muitas razões pera isso, em que nam falo por nã tornar isto á minha conta, mas com tudo toda a cousa de que nam sabiam dar rezão era dizer, he a noua Espanha."

Quant à la cartographie, mentionnons la carte de Petrus Roselli, de 1468, qui reproduit le groupe des Sept Cités (E. L. Stevenson, Facsimiles of Portulan Charts to the Hispanic Society of America (New York, 1916), $\mathrm{n}^{\circ}$ ii, cité par Morison, op. cit., 18; l'Atlas vénitien de 1489, au British Museum. Sur l'une de ces cartes, les Açores sont représentées presque correctement; les Sept Cités, au sud de l'île Flores; et Santa Ana, au nord de Corvo (Egerton Ms. 73). Plus tard, une carte également déposée aux archives du British Museum (Egerton Ms. 2803) datée des environs de 1508-1510, montre les Sept Cités au nord de l'Amérique; dans l'Atlas de Vaz Dourado, l'île légendaire apparaît également.

Voir l'étude remarquable d'Armando Cortesão, The Nautical chart of 1424, and the early discovery and cartographical representation of America, a study on the History of early navigation and cartography (Coïmbra, 1954), 70, paragraphe intitulé: "The Island of the Seven Cities."

Voir également l'étude récente de E. G. R. Taylor, professeur émérite de géographie de l'université de Londres, Imaginary Islands: a problem solved, "The Geographical Journal", (mars 1964), 105 et suiv., où l'on tente d'identifier les Sept Cités avec Terre-Neuve.

37 Jaime Cortesão, op. cit., II : 152.

38 Voyons ce que dit Las Casas (op. cit., $100 \mathrm{du}$ volume mentionné, qui comprend le chapitre XIII): “... Concuerda con esto lo que un marinero tuerto dijo al dicho Cristóbal Colon, estando en al puerto de Sancta Maria, que, en un viage que habia hecho à Irlanda, vido aquella tierra que los outros haber por alli creian é imaginaban que era Tartaria, que daba vuelta por el Occidente; la cual creo yo cierto que era la que ahora llamamos la de los Bacallaos, á la cual no pudieron llegar por los terribles vientos."

Ainsi, Las Casas et Colomb lui-même croyaient que cette terre des morues avait déjà été découverte bien avant le départ de ce dernier pour son fameux voyage. Il est encore évident que Morison ne croit pas à cette possibilité (op. cit., 27): "So Las Casas believed, and very likely Colombus believed, that Newfoundland had been sighted before his own first voyage. He did not sail in that direction, to be sure, but the report that land did exist beyond the Azores evidently gave him aid and comfort.

Should we then believe that Newfoundland was seen by this "third mariner" or someone else, before 1492 ? I think not."

Comme l'on est déjà d'accord sur le fait que Cabot découvrit le nord, Colomb le centre et, qui sait, Pinzon le sud, point n'est besoin d'étudier de nouveau la question avec plus de soin et d'impartialité ! Ce sont des slogans qui ont conquis les sympathies générales et se sont fixés à demeure dans la mémoire des hommes! Mais nous continuons à lutter contre ces formules toutes faites.

39 Velho Arruda, op. cit., 187: Alguns documentos..., 59; Morison, op. cit., 41.

40 Morison, op. cit., 45. 
41 Morison, op. cit., 47: "Estreito must provide two caravels fully equipped and victualled for six months, to be ready to sail on the first day of March, 1487, whilst Dulmo engages pilots and mariners for the fleet and pays their wages. Estreito will command one caravel and Dulmo the other, and "the German knight who is going with them" (Martin Behaim) may sail in either. And for forty days from their departure from Terceira, Dulmo shall be Captain General and set the course for both caravels and possess all lands discovered in that space of time, after which Estreito shall be Captain General and possess all other lands discovered thereafter and until their return to Portugal. All of which, and much more in the way of detail, the king confirmed on July 24 and August 4, 1486, promising that Estreito should enjoy all such privileges, liberties and franchises in his share of the discoveries as Dulmo had been granted originally."

Voir Alguns documentos...60 et suiv.; Velho Arruda, op. cit., 189 et suiv.; Joaquim Bensaude, Lacunes et surprises, 272; Las Casas, op. cit., 101, dans le passage suivant, semble faire allusion à Úlmo:

"... un marinero que se llamó Pedro de Velasco, gallego, digo al Cristóbail Colon en Múrcia, que, yendo aquel viage de Irlanda, fueron navegando y metiéndose tanto al Noroeste, que vieron tierra hácia el Poniente de Ibernia, y esta creyeron los que alli iban que debia de ser la que quiso discubrir un Hernan Dolinos..."

42 E. Brazão, op. cit., 59 et suivantes.

43 Harrisse, op. cit., 13 ; Drumond, op. cit., 68.

44 H. P. Biggar, Les Précurseurs de Jacques Cartier 1497-1534, collection de documents relatifs à l'Histoire primitive du Canada (Ottawa, 1913), "Ratification de la concession de terres faite par Gaspar Corte Real, à son frère Michel", dans l'original, en portugais, 67 et suiv.; traduction anglaise, 69 .

45 Harrisse, op. cit., 10.

46 Harrisse, op. cit., 39.

47 Harrisse, dans son œuvre remarquable, Découverte et évolution cartographique de Terre-Neuve et des pays circonvoisins - 1497-1501-1769, essais de géographie historique et documentaire (Paris, Londres, 1900), écrit: "Les documents n'attestent la présence d'aucun Européen à TerreNeuve avant Gaspar Corte Real. Dans l'état actuel de la question, l'historien impartial doit donc en attribuer la découverte aux Portugais. C'est aussi à ces habiles marins que revient le mérite d'avoir ébauché les premières cartes et la première nomenclature de cette île" ( $\mathrm{p}$. XXI de la préface). Et plus loin: "Au point de vue des documents aujourd'hui connus, c'est donc Gaspar Corte-Real qui découvrit l'île de Terre-Neuve. Pour prouver ce fait nous possédons de précieuses données. Ce sont les premiers dérivés portugais du prototype de la carte de Cantino. En les comparant entre eux et en relevant la nomenclature que porte la terre où, selon cette carte, Corte-Real atterrit après avoir quitté les abords du Groenland, on résout facilement la partie principale de la question" (38).

47a François 1er disait à l'Ambassadeur d'Espagne: “...passando camino y descubrir del ojo, no es adquerir possession" (Lettre de l'Ambassadeur d'Espagne à l'Empereur, le 27 Décembre 1540 - Archives Nationales de Paris, série $\mathrm{K}, 1485,1540-41$, $\mathrm{n}^{\circ} 41$; publiée par $\mathrm{H}$. P. Biggar, A Collection of Documents relating to Jacques Cartier and Sieur de Roberval (Ottawa, 1930), 170.

48 Giovanni Battista Ramusio, Terzo volume delle Navigationi et Viaggi (1565), 417 . 
49 Lopes de Gomara, Primera e segunda parte de la Historia general de las Indias (Saragoça, 1552), 177, dans l'édition de Vedia. de la note.

50 António Herrera, Decada I, liv. VI: 169; Harrisse, op. cit., 60

51 Jerónimo Osório, De rebus Emmanuelis Gestis (Lisbonne, 1571), liv. II : 84.

52 António Galvão, op. cit., édition de Burt Franklin, 96. Dans un document anglais de 1575 (?): A note of certayne navigations Hertoffore attempted for the discoveries of a passage through the straightes out of Northe sea into the south sea, publ. dans l'œuvre rééditée par Burth Franklin, de New York, "The three voyages of Martin Frobisher..." cité (p. 3), on lit: "Anno 1500. Moreover, in the yere of our Lord 1500, one Gaspar Corte reales, a pilot of Portingale from the northe parte of America was in these ilandes with two shippes, and brought with hym from thence threescore captyves of slaves."

53 Damião de Góis, Chronica do Felicissimo Rei Dom Manuel (Lisbonne, 1566), chap. LXVI: 65; (éd. de 1949), I: 146.

54 Dans Biggar, op. cit., 32 et suiv., dans l'original, en portugais; et 35 et suiv. de la traduction anglaise.

55 Dans Biggar, op. cit., 92 et suiv. dans l'original, en portugais; 94 et suiv. de la traduction anglaise.

56 Gago Coutinho, Gaspar Corte Real, communicação lida na sessão comemorativa dos Corte Reais, (tiré du "Boletim da Sociedade de Geografia de Lisboa", $\mathrm{n}^{\circ} 11$, nov. 1933) : D'après cet auteur, Gaspar aurait fait un voyage en 1499, effectuant à peu près le même parcours que celui de Colomb, mais plus au nord, jusqu'au Cap Breton. Cette reconstitution repose sur la carte de 1502 de Cantino, et qui n'est pas assez complète pour établir une date précise.

57 Harrisse, op. cit., 60.

58 Joaquim Verissimo Serrão, Historiografia Portuguesa (Lisbonne, 1962), 123.

59 Biggar, op. cit., XIV.

60 Harrisse, op. cit., 52 et suivantes.

61 A l'automne de 1502, Cantino, allant en Italie, apporta avec lui le document cartographique, et le remit au patricien Francesco Cataneo, à Gênes, qui fut ambassadeur de cette république en France et en Aragon, pour qu'il l'expédiât à Ferrare. De Rome, il écrivait cette lettre à Hercule d'Este:

"Ill.mo Principi et Ex.mo Duci et Domino Domino Herculi Duci Ferrarie et Domino meo Benefactorique observandissimo, Ferrarie.

Ill.mo Princeps et Ex.me Dux et Domine Domine mi observandissime etc. Per una de V. Ex in risposta d'una mia a giorni passati a quella drizata, ho inteso quanto in epsa se contiene, et maxime circha la Charta del navichare: Il che humilmente a V.S. respondendo adviso, che dicta Charta lassai in genoa a dicto messer Francesco Catanio et da lui hebbi Ducati vinte striti, cioè de libre tre ciascuno. Vera è che dicta Charta in portogallo a me de pacto facto mi costo Ducati dodici d'oro in oro. $\mathrm{Ma}$ astretto dal'bisogno et non havendo ove ricorrere fui sforzato a tuor dicti denari et far quanto a V.S. scrissi: la Charta è di tal sorte, et spero che in tel manera piacerà a V. Ex. che non gli serà molesto haver per epsa exbursato dicta quantidade, perchè di quel piu che sopra spenderà V.S. cioè de dodici. Si che V. Ex.tia piacendoli mi advisi quanto circha 
questo ho adoperare, et continuamente fra numero de suoi fideli Servitori mi tenga.

Romae die 19. novembris 1502.

Ill. et Ex. Dub. D. V. servitor Albertus Cantinus Scripsi. (archives de la Casa d'Este, Modena; publiée pour la première fois par Henry Harrisse, op. cit., en français et dans l'original, respectivement aux pages 70 et suivantes, et en appendice, document XXI: 215 et suivantes).

62 Jacques Chazelle, La Diplomatie (Paris, 1962).

63 Fernão Lopes de Castanheda, l'un des plus grands historiens portugais du XVIe siècle, écrivait dans son História dos Descobrimentos e Conquista da India Pelos Portugueses, I: 1 et suiv.:

"Antes que a India fosse descuberta pelos Portugueses, a mayor parte da especiaria, droga e pedraria dela se vazaua pelo mar roxo donde ya ter á cidade Delexandria, e ali a comprauão oa Venezianos que a espalhauão pela Europa, de que ho reyno de Portugal auia seu quinhão, que os Venezianos leuauão a Lisboa em garlés, principalmente reynãdo nos reynos de Portugal et Rey dõ João ho segundo deste nome: que como fosse de muyto altos pensamẽtos, e desejoso dacrecentar seus senhorios e emnobrecelos a seruiço do nosso señor, determinou de prosseguir ho descobrimento da costa de Guiné que seus antecessores tinhão comecado: porque por aquela costa lhe parecia q descobriria ho senhorio do Preste João das Indias de que tinha fama: per que por ali podesse entrar na India, donde per seus capitães podesse mandar leuar aquelas riquezas $q$ os Venezianos lhe yão vender. E coesta determinação mandou nouamente continuar este descobrimento per mar, per hũ Bertolameu diaz que foy almoxarife dos almazẽs de Lisboa, que mãdou por capitão mór a este descobrimento, em que descobrio aqle muyto grande e espantoso cabo dos antigos não conhecido: que agora se chama Cabo da boa Esperança...".

Morison, op. cit., 7, donne de ce passage la traduction anglaise de Lichefield, contemporain de la reine Elisabeth $\mathrm{I}^{\mathrm{re}}$. L'édition anglaise de cette œuvre est intitulée: The first Booke of the Historie of the Discouerie and Conquest of the East Indias... set foorth in the Portingale language by Hernam Lopes de Cantaneda. And now translated into English, by N. L., Gentleman (Londres, 1582). De cette époque également, nous avons une traduction italienne par Alfonso Ulloa, Historia dell'Indie Orientali, Scoperte, \& Conquistata da Portoghesi,... composti dal sig. Fernando Lopes di Castagneda (Venise, 1578). Et en 1553, une édition française sortit des presses de Michel Vascosan: Le premier livre de l'histoire de l'Inde; l'année suivante paraissait une nouvelle traduction: L'Histoire des Indes de Portugal, contenant comment l'Inde a esté decouverte... faict par Fernand Lopez de Castanhada, e traduict de Português em François par Nicolas de Grouchy (Anvers, 1554). Il y eut aussi une traduction espagnole. l'Europe.

On voit ainsi que cette œuvre causa une excellente impression sur

Verissimo Serrão écrit (op. cit., 103): “L'Historia, de Castanheda, connut une vie plutôt agitée. Elle comptait dix volumes, mais seulement huit ont été publiés. Les deux derniers n'ont pas reçu l'autorisation royale nécessaire à l'impression, à cause de l'influence exercée par certaines maisons nobles qui se croyaient atteintes dans leur prestige par l'omission ou le peu de relief que leur accordait Castanheda. Mais celui-ci ne voulut rien modifier à son manuscrit car, prétendait-il, l'historien doit suivre fidèlement la vérité historique." 
64 Nous sommes tout à fait d'accord avec le critère établi par António Sérgio dans sa préface remarquable à Obras Escolhidas, du Père António Vieira, dans la "Colecção de Classicos Sá da Costa".

De son côté, Launoy \& Vanderlinder, Expansion Coloniale des peuples européens - Portugal et Espagne (1907), 25 et suiv., écrivent: "Les Juifs dont l'activité contrastait vivement avec l'indolence des Portugais, étaient l'objet d'une réprobation générale... Grâce à la situation assez supportable qui leur était faite, surtout à partir du règne de Jean d'Aviz, les Juifs purent mettre au service de leur patrie d'adoption leurs aptitudes commerciales et leur activité. La plus grande partie, sinon tout le commerce extérieur du Portugal, était entre leurs mains. Ils jouissaient, sur toutes les places où ils trafiquaient, d'une haute réputation pour leur connaissance du commerce et leur intégrité. Dans aucun pays leur présence n'était plus utile qu'en Portugal, car ils possédaient précisément l'application et le sens des affaires qui manquaient aux autres classes de la population... Si l'on excepte les communautés juives, le Portugal était habité, au début du XVe siècle, par une race sobre et robuste, mais indolente et vaniteuse, presque dépourvue de toute culture scientifique ou littéraire."

Ricardo Jorge complète le tableau. Malgré les lueurs de vérité, l'âme hébraïque de cet écrivain portugais lui donne des tons exagérés; la prose recherchée qu'il emploie est tellement flamboyante qu'il nous donne toujours l'impression que nous sommes en train de lire une traduction d'un texte oriental, écrit de main judaïque: "... Ceux qui étaient absolument sans patrie en trouvaient une ici (au Portugal); ils incarnaient la quintessence de l'esprit patriotique de l'ancien Portugais le plus pur. Et cette foi transmet dans leur intégrité toutes les preuves: l'exil, le martyre et le bûcher." En est-il réellement ainsi? Il poursuit: "Déracinés et poursuivis, dépouillés de leurs biens et de leurs familles, ils saluaient alors leur terre natale, et, de père en fils, se transmettaient le pieux héritage."

"Et pourquoi? Une seule réponse est possible: parce qu'ils ont contribué à la grandeur et à la gloire de cette Patrie, autant ou davantage que ceux qui se considéraient ses vrais enfants. Comment ne pouvaient-ils pas la considérer comme la leur? "Celui qui a apporté son immense contribution, je l'ai déjà signalé, à nos expéditions de découvertes, personne ne le reconnaissait. Fût-il un Juif intelligent, illustre et actif, égal au chercheur et au savant, au négociant et à l'astronome, au financier et au mathématicien" (Ricardo Jorge, Origens e desenvolvimento da população do Porto (1897). "Dénué de tout esprit militaire, pacifiste comme on dirait aujourd'hui, son but était le développement de l'esprit de la richesse, la conquête économique et scientifique; ainsi, il devint le facteur intrinsèque de notre développement en tant que grand entrepôt et puissance mondiale. Et quand le fanatisme religieux, associé à l'envie locale contre ceux qui possédaient richesse et intelligence, écrasa cet échantillon particulier de la société portugaise, quand l'hystérie cléricale, le turpe pecus du parlement des trois pouvoirs et la politique imbécile éliminèrent l'élément noble, pensant et actif de la population nationale, notre Juif expulsé, fertilisant et enrichissant des terres d'exil (l'Angleterre et la Hollande), il déplaça avec lui l'axe du commerce et de la navigation, de la civilisation et du progrès." Ici, il semble que le commentaire de Ricardo Jorge est bien catégorique; ce qui n'est pas le cas lorsqu'il invoque, plus loin, le patriotisme profond qui accompagnerait le Juif expulsé et errant. Ricardo Jorge, Amato Lusitano, comentos à sua vida, obra $e$ época, ciclo Peninsular (Lisbonne s/d), éd. de "Instituto de Alta Cultura", 13 et suivantes. 
Voir la préface d'Arthur Moreira de Sá à l'œuvre de Diogo Lopes Rebelo, Do governo da República pelo Rei (De Republica gubernanda per Regem) (Lisbonne, 1951), éd. de "Instituto de Alta Cultura", paragraphe VI: XXIX et suiv., intitulé - "Os Judeus". (Les Juifs).

$65 \mathrm{La}$ lettre de Cantino à laquelle nous faisons allusion a été publiée par Biggar, op. cit., 61, dans son original en italien; à la page 63 dans sa version anglaise. Elle se trouve dans les "Archivio di Stato" à Modena, Dispacci della Spagna; Harrisse l'a publiée également, op. cit., 204 et suiv.; et dans les "Archivo dos Açores", IV : 424 et suiv.

La lettre de Pasqualigo à la Seigneurie de Venise, en date du 18 octobre 1501, se trouve à la Biblioteca Nacionali di S. Marco, Venise, Mss. Italiani Cl. 7, $\mathrm{n}^{\circ}$ 422, Diarii di Sanuto, IV: fol. 93; Harrisse l'a publiée, op. cit., 209 et suiv.; Les Archives des Açores, IV : 587 et suiv.; Biggar, op. cit., 65 dans le texte original italien; et à la page 66 de la version anglaise. Une autre lettre du 19 octobre de la même année, adressée à ses frères à Venise, a été publiée dans l'ouvrage Paesi nouamente retrouati (Vicenza, 1507), liv. VI: chap. CXXVI; aussi dans J. A. Williamson, The Cabot voyages and Bristol discovery under Henry VII (Cambridge, $1962)$, doc. 38: 229 et suiv.; sur les voyages de Gaspar Corte-Real, voir le même ouvrage, 118 et suiv.

${ }^{66}$ M. A. Buchanan, "Early Canadian History", Transactions of the Royal Society of Canada, XLII, series 111 (May 1948). Do Livro de Marco Paulo, édition portugaise de Valentin Fernandes (1502), réimpression dirigée par Francisco Maria Esteves Pereira, (édition de la Bibliothèque nationale de Lisbonne, 1922).

67 Edgar Prestage, op. cit., dans l'édition portugaise de 1943: 319 : “... D'après une lettre du cartographe Alberto Cantino, datée le 17 octobre dans cette ville, et selon laquelle les deux frères, sur le chemin du retour, n'ont pas aperçu de terre pendant quatre mois, il s'agit évidemment d'une erreur: ce sont des semaines au lieu des mois".

68 Morison, op. cit., 71.

$69 \mathrm{H}$. Harrisse, op. cit., 79, écrit: "Ce sont les perroquets qui produisirent une vive impression sur les navigateurs portugais, lors de la découverte du Brésil, qu'ils en firent le signe distinctif de cette contrée." Sur la carte dite de Cantino, le cartographe portugais qui a fait le dessin a illustré la partie nord du Brésil avec trois superbes perroquets rouge, vert et jaune.

70 Henry Percival Biggar (1872-1938) "historian and archivist, was born at the Carrying Place, Ontario, on August 9, 1872, the son of James Lyons Biggar and Isabel Hodgins. He was educated at the University of Toronto (B.A., 1894) and at the Oxford University (B. Litt., 1899; D. Litt., 1927). He entered the service of the Public Archives of Canada, and from 1905 to his death he was the chief archivist for Canada in Europe. He died at Worplesdon, Surrey, England, on July 25, 1938. He was the author of a treatise on The early trading companies of New France (Toronto, 1901), of which only 300 copies survived destruction by fire; and he edited The precursors of Jacques Cartier, (Ottawa, 1911), The works of Samuel de Champlain ( 6 vols, Toronto, The Champlain Society, 1922-36), The voyages of Jacques Cartier (Ottawa, 1924), and $A$ Collection of documents relating to Jacques Cartier and the Sieur de Roberval (Ottawa, 1930). In 1924 he was made an LL.D. of Queen's University, Kingston". W. Stewart Wallace, The Macmillan Dictionary of Canadian Biography (3e éd., 1963), 56.

Cette liste des cuvres de Biggar ne mentionne pas l'édition française, Les Précurseurs de Jacques Cartier 1497-1534, Collection de docu- 
ments relatifs à l'Histoire primitive du Canada (Ottawa, 1913), avec les documents dans les langues originales accompagnées de leur version anglaise. C'est cette édition que nous citons.

71 Biggar, op. cit., XVI de sa préface.

72 Milton Alexander Buchanan (1878-1952) "educationist, was born at Zurich, Ontario, on July 17, 1878. He was educated at the University of Toronto (B.A., 1901) and at the University of Chicago (Ph.D., 1906); and during the years 1902-04 he studied at Paris and Madrid. In 1906 he was appointed a lecturer in the department of Italian and Spanish in the University of Toronto, and he rose rapidly until he became head of the department in 1917. He became an Hispanic scholar with an international reputation; and in 1933 he was elected president of the Modern Language Association, the only Canadian ever elected to this position. In 1935 he was elected a fellow of the Royal Society of Canada. He retired from teaching in 1946, and he died at Toronto on May 2, 1952. He left his magnificent library of Spanish literature to the University of Toronto Library. He compiled a Graded Spanish wordbook (Toronto, 1927) : he was chairman of the committee that prepared Modern language instruction in Canada (2 vols., Toronto, 1928); and he edited Spanish poetry of the golden age (Toronto, 1942)." W. Stewart Wallace, op. cit., 90.

73 Publié dans les "Estudios Hispanicos: homenaje a Archer M. Huntington" (Wellesley, Mass., 1952). 